\title{
Nanoscale
}

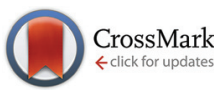

Cite this: Nanoscale, 2016, 8, 7672

\section{Polarized Raman scattering and SEM combined full characterization of self-assembled nematic thin films}

\begin{abstract}
R. Srikantharajah, K. Gerstner, S. Romeis and W. Peukert*
Elongated particles are predestined for a fast transfer of optical and electronical signals in a preferred direction, which is mandatory for a quick response in optoelectronic devices. The performance of the material is based on the quality of defect less alignment of the particles. On this account we present an easy non-invasive methodology for characterization of both surface and bulk order. The characterization of bulk order is performed by orientation dependent variation of the polarized Raman scattering signal on large areas by mapping. Scanning electron microscopy and image analysis on the surface complete the characterization. New insights in dip coated nematic structures clearly show the interplay of evaporation induced and shear-induced self-assembly and reveal a comprehensive mechanistic picture for nanorod assembly: the shear force dominated regime orients the particle in direction of withdrawal. At low withdrawal velocity, however, shear forces and evaporation counteract to produce a three-layered film where the top and bottom layers are oriented perpendicular to each other. The middle layer gives a clear evidence for a reorientation by convective flow.
\end{abstract}

Received 19th February 2016, Accepted 11th March 2016

DOI: $10.1039 / c 6 n r 01440 b$

www.rsc.org/nanoscale resulting film structure can be directly tuned by the withdrawal velocity. For dip coating of particles produced by sol-gel approaches, e.g. $\mathrm{TiO}_{2}, \mathrm{SiO}_{2}$ and for phospholipid films it is well known that three distinctly different deposition regimes exist, namely the capillary, the intermediate and the viscous drag regime, which depend on the withdrawal velocity. ${ }^{13,14}$ In biological shape-anisotropic systems, it was observed that Tobacco Mosaic Virus particles (TMV) are oriented from parallel to perpendicular on the surface by controlled evaporative self-assembly at the receding meniscus on a horizontal substrate by stick-slip motion. ${ }^{15,16}$ In biomimetic self-templating supramolecular structures, like phages, orient perpendicular in grooves and parallel in ridges due to a stick-slip motion in the capillary regime. ${ }^{17}$ The most popular theory for the selfassembly of shape anisotropic particles like nanorods or nanocylinders into anisotropic colloidal crystals is described by the Onsager theory. The theory holds for monodisperse spherocylinders with only hard-rod interactions. Based on the theory a transition from an isotropic to a nematic phase (where the spherocylinders have no positional order, but tend to point in the same direction) was predicted. The process is driven by maximization of entropy and is only valid for long rods with aspect ratios around $100 .{ }^{18}$ This theory holds for thermodynamically stable systems. At this point it is not clear, if this is also the case for the different regimes observed during dip coating: in the latter case the structure formation is a dynamic process. To assess the ordering of deposited films various
Institute of Particle Technology, Friedrich-Alexander-Universität Erlangen-Nürnberg, Cauerstraße 4, 91058 Erlangen, Germany.E-mail: wolfgang.peukert@fau.de 
experimental techniques have been used: 2D Fast Fourier transformation on the surface has been applied to analyse the angle distribution as measure for surface order. ${ }^{16,19-22}$ Polarization microscopy has been utilized to qualitatively characterize bulk orientation. ${ }^{21,23,24} \mathrm{X}$-ray and electron diffraction methods are commonly used to distinguish and quantify between vertical and horizontal bulk orientation in thin films. $^{25-29}$

Until now, however, the degree of order of horizontally orientated nanorods in thin nematic films has not been studied on the surface and in the bulk at the same time. In particular, a comprehensive study of shape anisotropic nanoparticles selectively deposited by dip-coating in the different deposition regimes has not been performed yet. For the different deposition regimes a unique combination of electron microscopy and image analysis on the surface and polarized Raman spectroscopy in the bulk reveals for the first time quantitative process-structure correlations. Moreover, for the first time the polarized Raman spectroscopy is introduced as a unique methodology for the characterization of oriented crystalline thin films made of uniaxial shape-anisotropic particles. $\mathrm{ZnO}$ promotes the shape-anisotropic growth to nanorods due to the wurtzite crystal structure. It is a promising candidate for applications in optoelectronic devices as solar cells, field effect transistors, Schottky diodes or light-emitting diodes. ${ }^{30-34}$ It has been shown recently that $\mathrm{ZnO}$ nanorods can be deposited into self-assembled dense thin films by standard methods like drop casting, spin coating or solvent evaporation from a vertically immersed substrate. ${ }^{20,23,35}$ In dip coating structure formation is triggered by the interplay of convective assembly in the capillary regime and shear forces in the intermediate and viscous drag regime. ${ }^{9,19,36-38}$ A flip from a parallel orientation of nanorods with respect to the contact line in the capillary regime to a perpendicular orientation of nanorods with respect to the contact line in the intermediate and viscous drag regime is observed. Interestingly, we observe for the first time a reorientation of the nanorods within the layer formed in the capillary regime. Based on these findings deposition and assembly mechanisms are proposed.

\section{Experimental}

\section{Preparation of $\mathrm{ZnO}$ nanorods}

Zinc oxide nanorods with a length of $144 \pm 63 \mathrm{~nm}$ and a diameter of $22 \pm 4 \mathrm{~nm}$ were obtained by precipitation from solutions of zinc acetate dihydrate $\left(\mathrm{ZnAc}_{2} \cdot 2 \mathrm{H}_{2} \mathrm{O}, \geq 99 \%\right.$ purity) and potassium hydroxide $(\mathrm{KOH}, \geq 85 \%$ purity) in methanol $(99.5 \%$ purity). ${ }^{39}$ All chemicals were purchased from Carl Roth $\mathrm{GmbH}$ \& Co KG (Karlsruhe, Germany) and were used without further purification. To $125 \mathrm{~mL}$ of zinc acetate dihydrate solution with a concentration of $0.71 \mathrm{~mol} \mathrm{~L}^{-1}$ an amount of $65 \mathrm{~mL}$ of $\mathrm{KOH}$ solution with a concentration of $1.4 \mathrm{~mol} \mathrm{~L}^{-1}$ was dropped within $10 \mathrm{~min}$ at a refluxing temperature of $65^{\circ} \mathrm{C}$. Subsequent refluxing of the suspension led to the formation and growth of ZnO nanorods. After the synthesis, the precipitate was washed twice to remove the remaining salt by centrifugation and subsequent redispersing of the precipitate in methanol.

\section{Preparation of well-stabilized dispersions}

The ZnO nanorods were transferred into absolute ethanol and were subsequently stabilized by adding $20 \mathrm{wt} \%$ (related to the ZnO mass) of TODA [2-(2-(2-methoxyethoxy)ethoxy)acetic acid] purchased from VWR (Darmstadt, Germany). To transfer the particles into ethanol the precipitate was washed four times by centrifugation and subsequent redispersions in ethanol. After addition of the stabilizer excess TODA was removed by the same procedure. ${ }^{23}$

\section{Preparation of $\mathrm{ZnO}$ nanorod thin films via dip-coating}

$\mathrm{ZnO}$ nanorod thin films were prepared with the dip-coater Nadetech ND-DC (Navarra, Spain). To control temperature and relative humidity the dip-coater was installed in a climate chamber (HPP110, Memmert, Germany): temperature sensors with deviations in the range of $\pm 1{ }^{\circ} \mathrm{C}$ and the relative humidity sensor with deviations in the range of $\pm 3 \%$ monitored the experiments. Pre-cleaned silicon wafer slides coated with $20 \mathrm{~nm} \mathrm{Ti,} 100 \mathrm{~nm}$ TiN and $500 \mathrm{~nm}$ Al were used as substrates. The substrates were assembled to the sample holder and were coated with $\mathrm{ZnO}$ nanorods in a three step process: first the substrate is immersed into the dispersion with a speed of $150 \mathrm{~mm} \mathrm{~min}{ }^{-1}$. Then a hold time of $180 \mathrm{~s}$ is set to equilibrate the contact line (liquid-air-substrate interface). As a final third step the substrate is withdrawn from dispersion at a constant speed between $0.5 \mathrm{~mm} \mathrm{~min}{ }^{-1}$ and $150 \mathrm{~mm} \mathrm{~min}^{-1}$.

\section{Characterization of the $\mathrm{ZnO}$ nanorod suspension}

The particle size distributions of the $\mathrm{ZnO}$ nanorod synthesis were characterized by a field emission scanning electron microscope (SEM, ULTRA 55, Carl Zeiss NTS GmbH, Oberkochen, Germany). The SEM samples were prepared by dropping the dispersions on $\mathrm{Si}$ wafer slides that were cleaned in absolute ethanol. For each sample the lengths and the diameters of individual rods (up to 500) were measured by using the software Image from the National Institutes of Health (Texas, USA). To qualify primary particle stabilization the diluted dispersions $(0.01 \mathrm{wt} \%)$ were analyzed by dynamic light scattering (DLS) using a Zetasizer ZEN3600 (Malvern Instruments, Worcestershire, UK).

\section{Characterization of $\mathrm{ZnO}$ nanorod thin films}

The substrate is positioned horizontally for SEM and Raman analysis after processing vertically during dip-coating. The quantitative characterization of the dip-coated films regarding surface order and surface porosity was conducted by a combination of SEM and ImageJ. To determine the nanorods alignment in the film, the angle of at least 600 nanoparticles in three independent images were analyzed for each sample. The relative frequencies of different angles to a common reference axis was measured. To estimate and minimize any statistical influences up to 2000 nanoparticles were analyzed for selected samples. To exclude effects of the substrate edge and the preliminary contact 
line SEM images were taken only from the center of the dipcoated films. Additionally, the surface porosity (SP) was evaluated by adjusting contrast and brightness to sharpen the edges between pores and particles. Representative interpretation is only possible at given constant film thickness and homogeneity. Film thickness and the route mean square (rms, roughness) were measured by a tactile profilometer (DektakXT, Bruker Nano Surfaces Division, Karlsruhe, Germany). Each sample was screened along three lines in direction of the substrate withdrawal. Bulk order is analyzed by polarized and orientation-dependent Raman spectroscopy. Raman spectra were recorded for Raman shifts between 175 and $475 \mathrm{~cm}^{-1}$ using a confocal Raman spectrometer (LabRAM HR Evolution, Horiba, Dresden, Germany). A He-Ne laser $(\lambda=633 \mathrm{~nm})$ was used as the excitation source. The laser beam was focused on the thin film with a 100fold objective (numerical aperture 0.9 , laser spot size $\approx 0.85 \mu \mathrm{m}$ ). Due to the $\mu \mathrm{m}$-size laser spot the resolution is limited to a $\mathrm{ZnO}$ nanorod ensemble covering an average area of minimum $850 \mathrm{~nm}$ in diameter. The hole and slit were fully opened at $1200 \mu \mathrm{m}$ to record the total signal intensity over the bulk film. The grating was set at $1800 \mathrm{gr}$ per $\mathrm{mm}$. The polarization was realized by using a half-wave plate so that the incident light polarization can be rotated by an angle $\Theta$. The scattered light passes through an analyzer to efficiently detect the phonons polarized parallel or perpendicular with respect to the optical axis of the sample. A scrambler is installed to remove polarization effects which are caused by the diffraction grating by transforming the linear polarization into a circular polarization. Maps of the size of $20 \mu \mathrm{m} \times 20 \mu \mathrm{m}$ were analyzed with a step size of $1.7 \mu \mathrm{m}$ (169 points) to visualize the order in the bulk films.

\section{Results and discussion}

\section{Dip-coating of colloidal dispersions of shape anisotropic particles}

In our study controlled self-assembly of $\mathrm{ZnO}$ nanorods into nematic structures is achieved by dip coating. The substrate is withdrawn from bulk dispersion at constant velocity. The deposition process is conducted in a climate chamber $\left(40{ }^{\circ} \mathrm{C}, 10 \% \mathrm{r}\right.$. $\mathrm{H})$. The single stabilized nanorods in the bulk dispersion (20 wt\%) have an aspect ratio of 6.5. From literature for spherical particles and sol-gel solutions it is well known that three distinct regimes are applicable to tune the film thickness by dip-coating. ${ }^{13,40,41}$ The first regime at very low withdrawal velocities is described as capillary regime, evaporation regime or gravity driven stick-slip regime depending on the focus of the studies (see Fig. 1 I). Independent of the naming, in this regime universal validity is given for two aspects. One is the initial contact line between substrate and liquid, which is curved resulting in a lower capillary pressure and increasing vapour pressure, so that the evaporation rate is enhanced at the contact line. The evaporation induces a convective liquid flow, where the dispersed particles are transported and accumulated at the contact line. The second important aspect is that the contact line is dynamically moving on the substrate by a

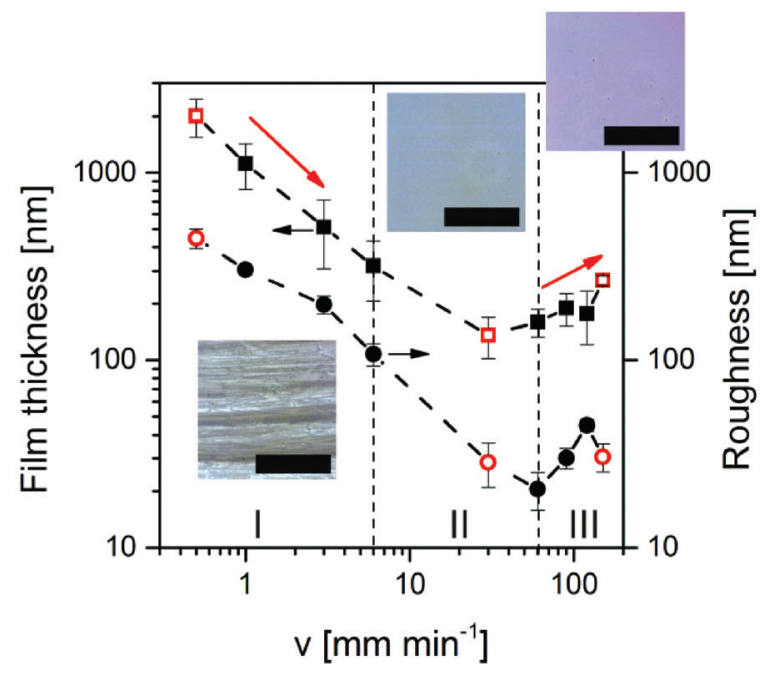

Fig. 1 Film thickness and roughness trend over the withdrawal rate of $0.5 \mathrm{~mm} \mathrm{~min}^{-1}$ to $150 \mathrm{~mm} \mathrm{~min}^{-1}$. Film thickness decrease in the capillary regime (I), lowest thickness in the intermediate regime (II) and increase in film thickness in the viscous drag regime (III) with increasing withdrawal rate. Insets in each of the regimes depict optical microscope images of the red highlighted data points. Stick-slip motion results in rough surfaces, while smooth surfaces with low roughness are analyzed in the intermediate and viscous drag regime. Scale bar represents $500 \mu \mathrm{m}$.

pinning and unpinning mechanism. In dip-coating this stickslip effect is externally controlled by the meniscus moving rate by withdrawing the substrate from bulk dispersion at very low withdrawal rates. Ghosh et al. and Bodiguel et al. have shown this for spherical particles, while Huang et al. has shown it for nanowires. ${ }^{38,42,43}$ In these studies they describe that substrate withdrawal stretches the meniscus until it cannot be retained by the network of deposited particles at the contact line. Watanabe et al. describe the meniscus stretch as a concave surface against the substrate. The contact line has to jump and find a new equilibration position. For non-rigid single-wall carbon nanotubes Li et al. describe the contact line movement as a dynamical pinning and kink-induced zipping. ${ }^{38,42-45}$ This periodic movement of the meniscus results in stripes in the deposited layer. Stripe like patterns, as described, are also visible in our study for the capillary regime as depicted in the optical microscope image in Fig. 1 (I) for a ZnO nanorod sample withdrawn at $0.5 \mathrm{~mm} \mathrm{~min}^{-1}$ (red highlighted data). The mean film thickness is about $2 \mu \mathrm{m}$. However, in contrast to spherical particles rod-like particles have a higher contact area and higher affinity to the substrate, so that a planar coverage is possible. This results in $\mathrm{ZnO}$ nanorod deposition in the stripe and in the spacing; this is not always the case for spherical particles. ${ }^{44}$ In this regime increasing the withdrawal rate results in thinner films due to decrease of the pinning force as commonly known. ${ }^{3}$ The evaporation rate is constant while the withdrawal rate is increasing, so the particle deposition rate to the contact line is lower and the film thickness decreases. At high withdrawal velocities the effect on film thickness is reversed. The film thickness increases with increasing withdrawal rate (see Fig. 1 
III). This regime is commonly known as viscous drag or Landau-Levich regime. ${ }^{13}$ The contact angle is reduced to a sufficiently low value that the contact line is pinned and a thin film is entrained on the substrate. ${ }^{42,46}$ In this regime gravity induced viscous drag is predominant and the film thickness depends on the density, the surface tension and the viscosity of the dispersion. With higher withdrawal rates a higher amount of particles is entrained from the bulk dispersion which results in thicker films. Increasing the particle concentration enhances viscosity and the film thickness increases at high withdrawal rate. The effects in the capillary and viscous drag regime are counterbalanced in the intermediate or transition regime resulting in a minimum film thickness. The optical microscope image in Fig. 1 II depicts a homogeneous surface of the sample in the intermediate regime comparable to the viscous drag sample. The color difference in both samples is caused by the film thickness, since the viscous drag sample withdrawn at $150 \mathrm{~mm} \mathrm{~min}^{-1}$ has a mean thickness of $266 \mathrm{~nm}$ and the intermediate sample withdrawn at $30 \mathrm{~mm}$ $\min ^{-1}$ has a minimum mean thickness of $136 \mathrm{~nm}$. The film thickness from capillary over intermediate to viscous drag regime is resulting in a parabolic trend. Our data fit well with literature for the critical velocities for spheres from capillary to intermediate regime at about $6 \mathrm{~mm} \mathrm{~min}^{-1}$ and from intermediate to viscous drag regime at about $60 \mathrm{~mm}$ $\min ^{-1} \cdot{ }^{13,14,41,43}$ The film roughness is highest in the capillary regime due to the stick-slip effect and lowest in the intermediate and viscous drag regime due to the homogeneous drying of the entrained film. The main goal is to gain reproducible nematic thin films with adjustable film thicknesses and homogeneity by dip-coating. The film thickness is tunable by the withdrawal rate, but the quality of nematic order has to be investigated in detail. In the following SEM-based image analysis on the surface and polarized Raman spectroscopy in the bulk reveal quantitative process-structure correlations.

\section{Quantification of surface order via SEM-based image analysis}

SEM imaging as shown in Fig. 2 reveals the nematic order of the thin films in all three regimes (red marked data points of Fig. 1). The capillary regime sample shows densely packed ZnO nanorods orientated predominantly parallel to the contact line (see Fig. 2a). In Fig. 2b the intermediate sample is
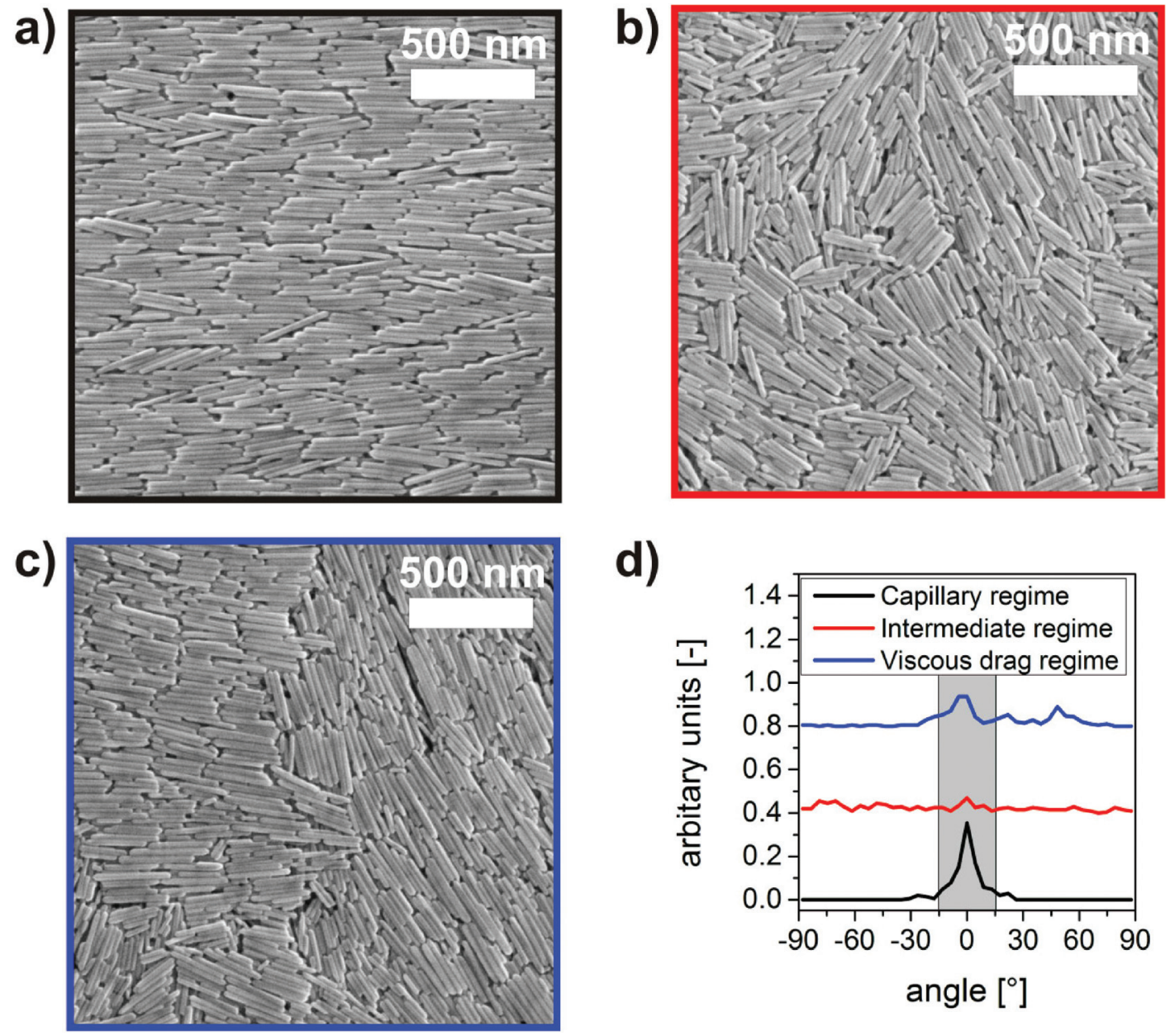

Fig. 2 SEM images of samples from the capillary regime (a), from the intermediate regime (b) and viscous drag regime (c). Angle distribution of samples (a)-(c) is depicted in (d) in order to determine the surface order by relating the integrated area between $+15^{\circ}$ to $-15^{\circ}$, to the integrated area between $+90^{\circ}$ to $-90^{\circ}$. 
shown with major orientation of the nanorods perpendicular to the contact line. Qualitatively there is less order on the surface. In the viscous drag regime the $\mathrm{ZnO}$ nanorod film shows two major domains. One with parallel orientation to the contact line and one with a perpendicular orientation (see Fig. 2c). By screening the angle distribution of the nanorods in the images an easy access to the surface order of nematic thin films is given. To this end the measured angles $(\theta)$ of the individual rods were normalized in between $+90^{\circ}$ and $-90^{\circ}$. The maximum frequency in the angle distribution was set to zero. For each sample the area in the range of $+15^{\circ}$ to $-15^{\circ}$ in the resulting angle distribution was integrated and compared to the integrated area in the range of $+90^{\circ}$ to $-90^{\circ}$. The ratio between both areas is defined as the degree of surface order (SO - see eqn (1)).

$$
\mathrm{SO}=\frac{\int_{-15^{\circ}}^{15^{\circ}} f(\theta) \mathrm{d} \theta}{\int_{-90^{\circ}}^{90^{\circ}} f(\theta) \mathrm{d} \theta}
$$

The angle distribution of the samples is illustrated in Fig. 2d. The grey shaded area in the distribution curves shows the integrated area between $+15^{\circ}$ to $-15^{\circ}$, which was related to the integrated area between $+90^{\circ}$ to $-90^{\circ}$ for calculating the SO. The angle distribution in the capillary regime depicts one distinct peak with relative narrow deviation, resulting in high surface order of $86 \%$. In the intermediate regime the angle distribution does not show a distinct peak and is broad and flat. No common orientation direction is visible. The surface order is about $31 \%$. In the viscous drag regime the angle distribution shows two major broad peaks. Conclusively two main domains exist. The grey shaded peak shows a surface order of $46 \%$. In all samples the mean surface porosity ranged between 4 to $12 \%$. Consequently in all regimes dense $\mathrm{ZnO}$ nanorod films are produced. Mean values with deviations are obtained for SO and SP by analyzing at least three positions on the $\mathrm{ZnO}$ nanorod film.

\section{Quantification of bulk order via polarized Raman scattering}

Easy access to the surface order is obtained by analyzing the angle deviation of each $\mathrm{ZnO}$ nanorod in a thin film. Access to bulk order is possible through breaking the thin film including the substrate, so that the cross section of the film is revealed in SEM. The disadvantage of this invasive method is the breaking, which is not always smooth over the whole film. $\mathrm{ZnO}$ nanorods can be peeled off at the cross section, and it is difficult to distinguish between the planes, i.e. the bulk order over the film height is not accessible.

Therefore polarized Raman scattering is proposed as an additional non-invasive bulk order characterization method in this study. Distinct discrimination of a nanorod ensemble oriented parallel or perpendicular is possible by polarizationdependent scattering of the incident laser beam, which has unique properties dependent on the orientation of a nanorod ensemble in an anisotropic crystalline structure. Raman activity is given, if a change in the polarizability of a phonon in a crystal structure is evident. If a Raman active material is grown as a shape anisotropic particle with one crystal axis different from the other two axes and if predominantly all these particles are aligned along a main axis the bulk order can be characterized. Due to the anisotropy in the interatomic forces the atoms are displaced differently by parallel or perpendicular incident beam. ${ }^{47}$ Defined shapes of elongated particles as rods, wires or ellipsoids made of e.g. ZnO, ZnS, SiC, GaAs, GaN, CdS show polarization dependent Raman scattering. ${ }^{4-52}$ Often metal oxides are rather weak Raman scatterers. Therefore limitations due to a weak Raman signal are given if the films are too thin.

In particular, the wurtzite $\mathrm{ZnO}$ has 12 phonon modes consisting of 9 optical modes and 3 acoustic modes and belongs to the space group $C_{6 \mathrm{v}}{ }^{4}$ in the Schoenflies notation. ${ }^{53}$ From group theory four Raman active modes $\mathrm{A}_{1}+\mathrm{E}_{1}+\mathrm{E}_{2}{ }^{\text {low }}+\mathrm{E}_{2}$ high are expected. Both $\mathrm{E}_{2}$ modes are associated with the vibration of the heavy $\mathrm{Zn}$ sub-lattice and the motion of oxygen atoms respectively. $\mathrm{A}_{1}$ and $\mathrm{E}_{1}$ modes are polar modes and split into transverse optical (TO) and longitudinal optical (LO) phonons due to the hexagonal structure and its anisotropy. In theory it is shown that phonons parallel to the $c$-axis of the nanorod enhance the intensity of the $\mathrm{A}_{1}(\mathrm{TO})$ at about $378 \mathrm{~cm}^{-1}$ and phonons perpendicular to the $c$-axis allow $\mathrm{A}_{1}(\mathrm{TO})$ and $\mathrm{E}_{2}{ }^{\text {high }}$ at about $438 \mathrm{~cm}^{-1} .^{54}$ Theoretically only $\mathrm{E}_{1}(\mathrm{TO})$ between $413-420 \mathrm{~cm}^{-1}$ is allowed in cross polarization. But due to defects, meaning zinc and oxygen vacancies it is possible that $\mathrm{A}_{1}$ (TO) and $\mathrm{E}_{2}{ }^{\text {high }}$ are also visible in cross polarization. ${ }^{52}$ Moreover, $\mathrm{E}_{1}$ (TO) can be visible, when the $\mathrm{ZnO}$ nanorod ensemble is not perfectly aligned. For example when the ensemble is perpendicularly oriented to a parallel polarization so that a pseudo cross polarization is evoked. Chien et al. and Yu et al. have shown polarized Raman scattering on a $\mu \mathrm{m}$ sized single ZnO rod. ${ }^{51,52}$ The optical phonon confinement in $\mathrm{ZnO}$ nanoparticles show under resonance conditions second order Raman spectra arising from zone-boundary phonons 2-TA(M), $2-\mathrm{E}_{2}(\mathrm{M}){ }^{53,55}$ In our bulk order studies $\mathrm{A}_{1}(\mathrm{TO}), \mathrm{E}_{1}(\mathrm{TO})$ and $\mathrm{E}_{2}{ }^{\text {high }}$ modes are relevant to distinguish between a primarily perpendicular or parallel orientation of the $\mathrm{ZnO}$ nanorod ensemble as explained before. Therefore the Raman spectrum is acquired in the range of $175 \mathrm{~cm}^{-1}$ and $475 \mathrm{~cm}^{-1}$. All relevant phonon mode frequencies of wurtzite $\mathrm{ZnO}$ in the measured range are in summary: 2-TA(M) at $203 \mathrm{~cm}^{-1}, 2-\mathrm{E}_{2}(\mathrm{M})$ at $331 \mathrm{~cm}^{-1}, \mathrm{~A}_{1}$ (TO) at $378 \mathrm{~cm}^{-1}, \mathrm{E}_{1}(\mathrm{TO})$ between $413-420 \mathrm{~cm}^{-1}$ and $\mathrm{E}_{2}{ }^{\text {high }}$ at $438 \mathrm{~cm}^{-1}$.

The Porto notation $A(B, C) D$ is used to describe the Raman geometry and polarization where $A$ is the direction of the propagation of the incident light, $B$ the direction of the polarization of the incident light, $C$ the direction of the polarization of the scattered light and $D$ the direction of the propagation of the scattered light. Accordingly $A$ and $D$ were set on the $Z$ axis and the initial contact line on the thin film is set as $X$ axis and the substrate withdrawal direction is set as $Y$ axis. Two different polarization configurations $Z^{\prime}(X, X) Z, Z^{\prime}(Y, Y) Z$ were investigated on the same position of the nematic $\mathrm{ZnO}$ nanorod thin films. After baseline correction the spectra were normalized to $2-\mathrm{E}_{2}(\mathrm{M})$ at $331 \mathrm{~cm}^{-1}$ since this peak is indifferent to polarization. The spectra were fitted by Lorentzian shape to profiles at $\mathrm{A}_{1}(\mathrm{TO}), \mathrm{E}_{1}(\mathrm{TO})$ and $\mathrm{E}_{2}{ }^{\text {high }}$. For quantification of the 
bulk order over the film a bulk order factor (BO) is introduced, where the integrated band intensity of $\mathrm{A}_{1}(\mathrm{TO})$ is compared to the sum of all other relevant modes. As described before, phonons parallel to the $c$-axis of the $\mathrm{ZnO}$ nanorod enhance the intensity of the $\mathrm{A}_{1}(\mathrm{TO})$ at about $378 \mathrm{~cm}^{-1}$.

$$
\mathrm{BO}=\frac{I_{\mathrm{A}_{1}(\mathrm{TO})}}{I_{\mathrm{A}_{1}(\mathrm{TO})}+I_{\mathrm{E}_{1}(\mathrm{TO})}+I_{\mathrm{E}_{2}} \text { high }}
$$

At very low withdrawal rate in the capillary regime the layer seems to be internally highly disordered: a one-dimensional, in-plane, nematic ordering as observed on the surface (see Fig. 3a) is not given. Qualitatively only the top layer reveals a nematic order parallel to the contact line in the cross section. The bottom layer shows an in-plane orientation of the nanorods rotated by $90^{\circ}$ compared to the top layer. In this layer the nanorods are oriented perpendicular to the contact line or parallel to the withdrawal direction. In between a nanorod re- orientation connecting the top and bottom layer is revealed, which is observed for the first time to our knowledge. At a first glance, the overall bulk film looks disordered. Therefore it is expected that $Z^{\prime}(X, X) Z$ and $Z^{\prime}(Y, Y) Z$ configurations will not show a distinct phonon polarization. Consequently Fig. $3 \mathrm{~b}$ shows all three modes $\mathrm{A}_{1}(\mathrm{TO}), \mathrm{E}_{1}(\mathrm{TO})$ and $\mathrm{E}_{2}{ }^{\text {high }}$ in both configurations. In Fig. 3c the Raman map for the configuration $Z^{\prime}(Y, Y) Z$ in direction of substrate withdrawal is depicted showing a mean $\mathrm{BO}$ of $19 \pm 3 \%$. In $Z^{\prime}(X, X) Z$ configuration a mean BO of $29 \pm 4 \%$ is calculated. This reveals that in total more phonons are parallel polarized to the $c$-axis in the $Z^{\prime}(X, X) Z$ configuration, i.e. overall more nanorod ensembles are oriented parallel to the contact line. Qualitatively this is confirmed in the SEM image in Fig. 3a.

The results obtained for the intermediate sample are shown in Fig. 4. The cross-sectional SEM image in Fig. 4a reveals predominant orientation of $\mathrm{ZnO}$ nanorods parallel to the withdrawal direction, respectively along the $Y$ axis. Consequently the $c$ -

a)

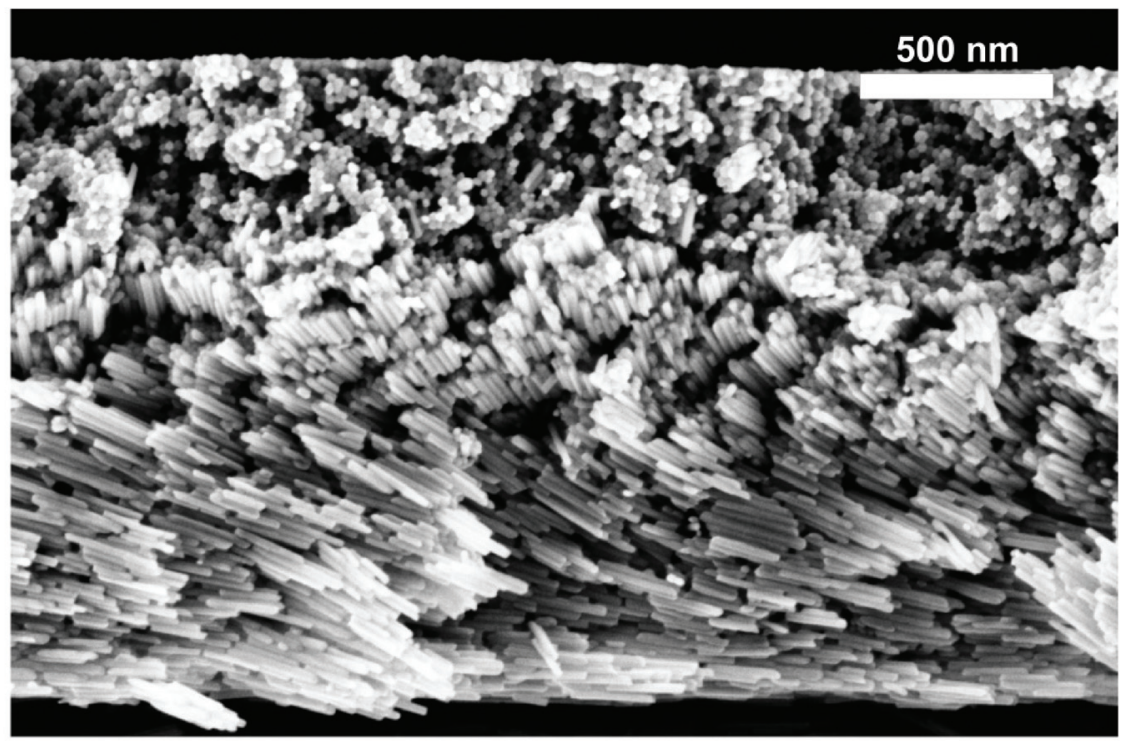

b)

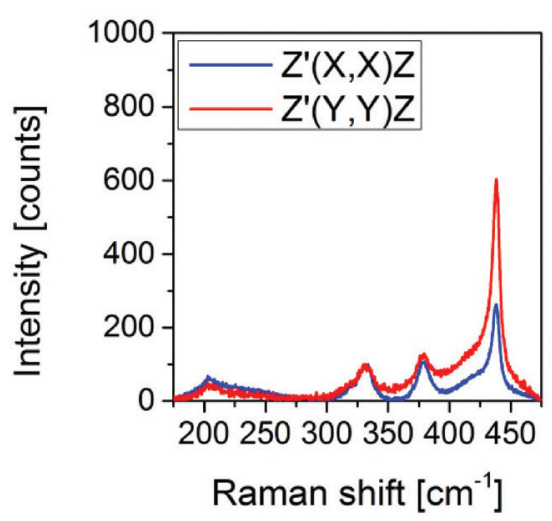

C)

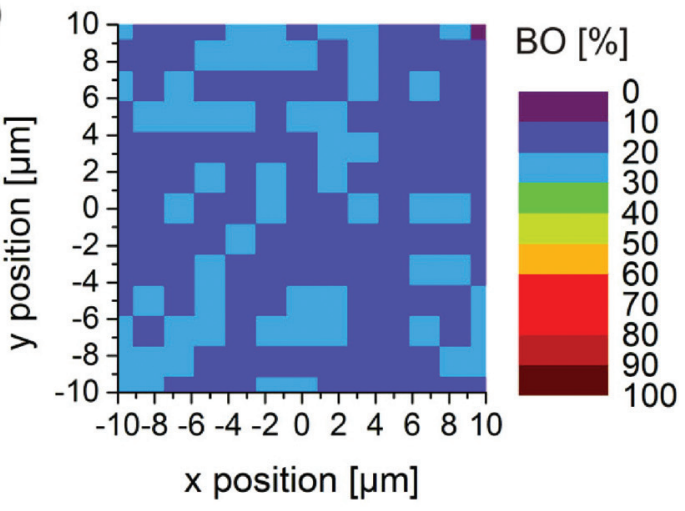

Fig. 3 SEM image of the cross section of a sample processed in the capillary regime (a). Single spectrum of the capillary film polarized in $Z^{\prime}(X, X) Z$ (parallel to the initial contact line) and $Z^{\prime}(Y, Y) Z$ (parallel to the substrate withdrawal direction) configuration (b). Raman map calculating the bulk order factor in $Z^{\prime}(Y, Y) Z$ configuration (c). 


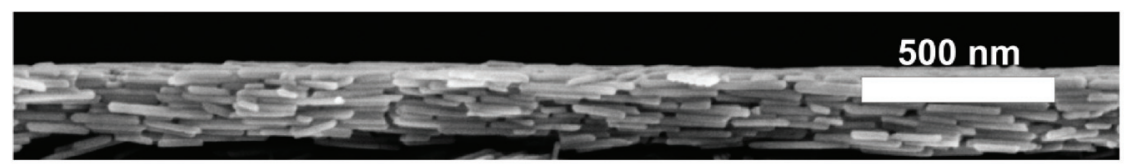

b)

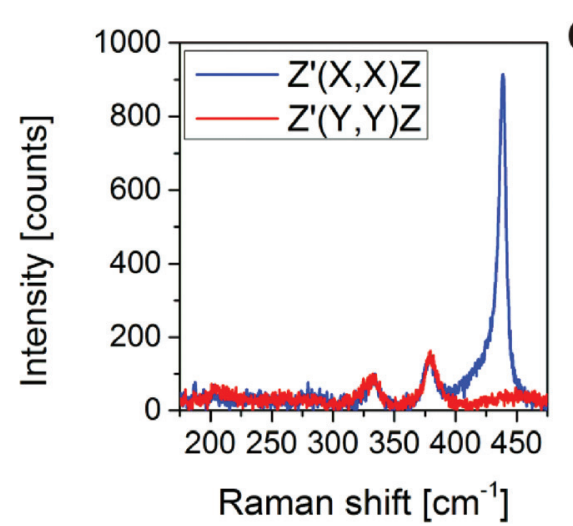

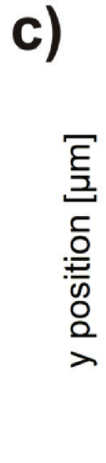

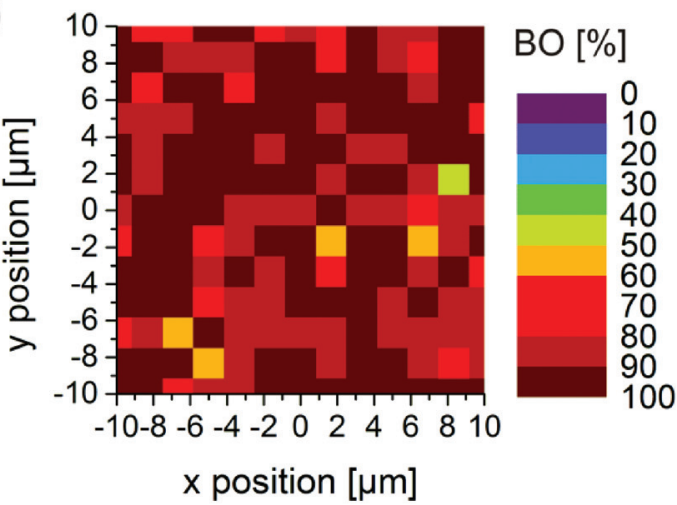

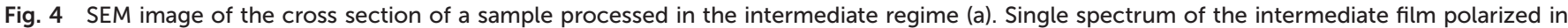

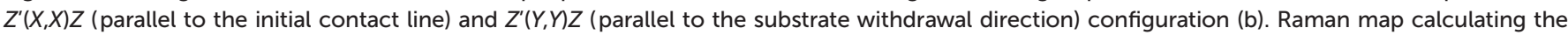
bulk order factor in $Z^{\prime}(Y, Y) Z$ configuration (c).

axis of the nanorods is oriented along the $Y$-axis. Hence, in the intermediate regime it is expected that the intensity of the $\mathrm{A}_{1}$ (TO) is enhanced at $Z^{\prime}(Y, Y) Z$ polarization, while in contrast at $Z^{\prime}(X, X) Z$ the phonons are perpendicular polarized to the $c$-axis so that $\mathrm{A}_{1}$ (TO) and $\mathrm{E}_{2}{ }^{\text {high }}$ are visible. In Fig. $4 \mathrm{~b}$ the single spectra in both configurations meet the expectations. A deviation of nanorod ensemble orientation from the withdrawal direction is depicted in Fig. 4c in form of the BO as Raman map. The mean BO is $88 \pm 9 \%$, which is very high. Moreover, over the map area a high homogeneity in bulk orientation is achieved. The comparison of the same sample regarding the surface order reveals also a predominant orientation of the nanorods perpendicular to the contact line and parallel to the withdrawal direction (see Fig. 2b). However, in contrast to the capillary regime an overall homogeneous bulk order is achieved in the intermediate regime.

In Fig. 5 SEM and Raman analysis of the sample processed in the viscous drag regime is depicted. The cross-sectional SEM image in 5 a reveal predominant orientation of $\mathrm{ZnO}$ nanorods parallel to the withdrawal direction, respectively along the $Y$ axis as in the intermediate regime. Consequently the single spectra in both configurations led to the expectation of similar evolution of the Raman modes $\mathrm{A}_{1}(\mathrm{TO}), \mathrm{E}_{1}(\mathrm{TO})$ and $\mathrm{E}_{2}{ }^{\text {high }}$. In Fig. $5 \mathrm{~b}$ the single spectra in both configurations confirm this. The mean BO extracted from the Raman map is around $70 \pm 12 \%$. The map itself shows a more inhomogeneous orientation over the whole film (see Fig. $5 \mathrm{c}$ ), while the surface order in the viscous drag sample has a higher surface order compared to the intermediate sample. Also the single spectra in Fig. 5b shows a non-perfect orientation due to nonvanishing $\mathrm{E}_{2}{ }^{\text {high }}$ mode in the $Z^{\prime}(Y, Y) Z$ configuration.

\section{Comparison of surface and bulk order}

The dissenting orders on the surface and in the bulk for the three samples from the capillary, intermediate and viscous drag regime justify that a more detailed comparison between surface and bulk order on nematic ZnO nanorod films has to be conducted.

In Fig. 6 the surface order and the bulk order are plotted against the withdrawal rate in the range of $0.5 \mathrm{~mm} \mathrm{~min}^{-1}$ to $150 \mathrm{~mm} \mathrm{~min}^{-1}$. The surface order decreases with withdrawal rate in the capillary regime (I) and increases with higher withdrawal rate in the intermediate (II) and viscous drag regime (III). Bulk order shows a reversed trend, where in the capillary regime the bulk order is low and is at maximum in the intermediate regime. In the viscous drag regime the bulk order is slightly decreasing again. Both trends are explained in the following by the structure formation mechanism.

\section{Structure formation mechanism}

From our investigations it is obvious that surface order alone does not give a full characterization of the $\mathrm{ZnO}$ nanorod thin film. Although the substrates are just withdrawn from the bulk dispersion with varying velocities the thin films reveal different structures.

Therefore it is clear that different structure formation mechanism are at the bottom of the capillary or gravity driven stick-slip regime compared to the viscous drag or LandauLevich regime. As discussed in the previous section, in the capillary regime the evaporation rate $(J)$ is enhanced at the contact line resulting in a convective liquid flow, where the dispersed nanorods are transported and accumulated at the 
a)

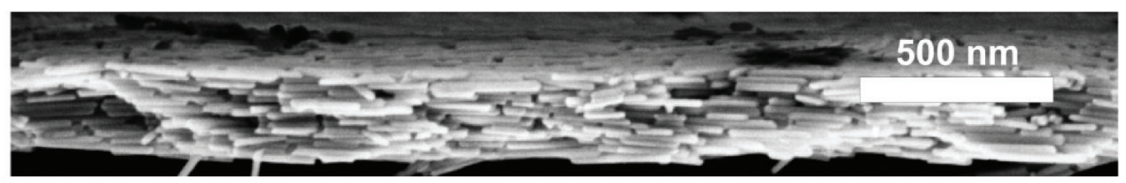

b)

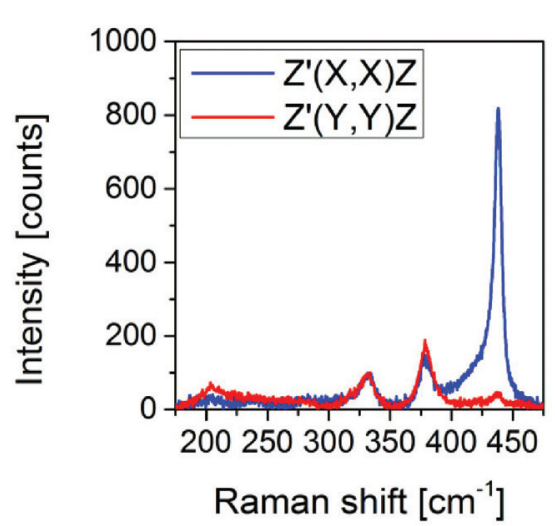

c)

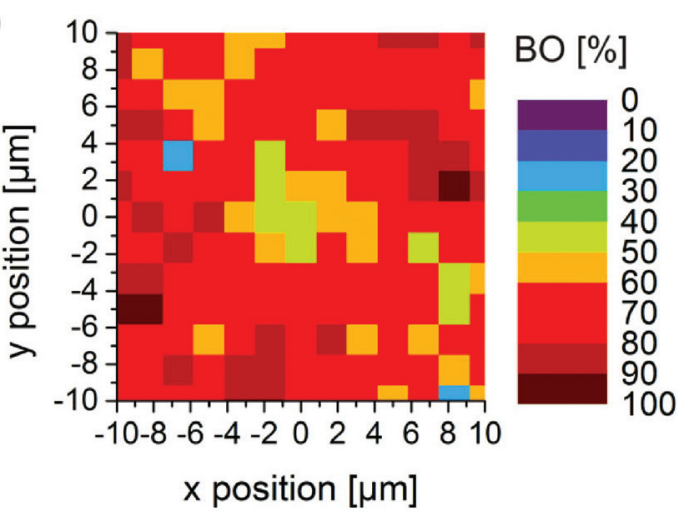

Fig. 5 SEM image of the cross section of a sample processed in the viscous drag regime (a). Single spectrum of the viscous drag film polarized in $Z^{\prime}(X, X) Z$ (parallel to the initial contact line) and $Z^{\prime}(Y, Y) Z$ (parallel to the substrate withdrawal direction) configuration (b). Raman map calculating the bulk order factor in $Z^{\prime}(Y, Y) Z$ configuration (c).

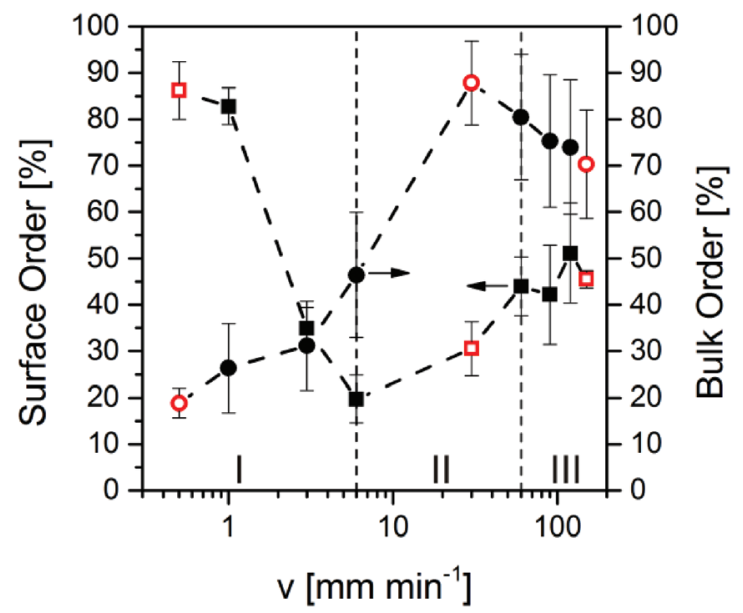

Fig. 6 Surface and bulk order trend over the withdrawal rate of $0.5 \mathrm{~mm}$ $\mathrm{min}^{-1}$ to $150 \mathrm{~mm} \mathrm{~min}^{-1}$. Surface order decreases with withdrawal rate in the capillary regime (I) and increases with higher withdrawal rate in the intermediate (II) and viscous drag regime (III). Bulk order shows a reversed trend, where in the capillary regime the bulk order is low and is at maximum in the intermediate regime. In the viscous drag regime the bulk order is slightly decreasing again.

contact line. By externally controlling the meniscus moving rate by withdrawing the substrate from bulk dispersion at very low withdrawal rates, the contact line is dynamically moving on the substrate. This withdrawal velocity $(v)$ and gravity-driven $(g)$ periodic movement of a meniscus result in stripes. Firstly, the particles close to the substrate surface are oriented in direction of withdrawal due to shear forces at the boundary where the withdrawal direction counter acts the gravitational force on the withdrawn meniscus, which is adhered to the substrate. The induced shear force will be at maximum near the substrate surface. This evokes an in-plane orientation of the $\mathrm{ZnO}$ nanorods parallel to the withdrawal direction. Secondly, the individual $\mathrm{ZnO}$ nanorods in the wet film are transported to the contact line by the solvent evaporation flux, where they maintain their rotational and diffusional freedom. The $\mathrm{ZnO}$ nanorods transported to the top of the wet film are oriented parallel to the contact line in order to maximize the interfacial coverage per particle which in turn minimizes the Helmholtz free energy of the system. This is mainly due to the evaporation induced convective assembly on the liquid surface. The low withdrawal velocity and evaporation induce a wavelike motion of the wet film containing the $\mathrm{ZnO}$ nanorods. The nanorods follow this wavelike motion, so that a reorientation of the nanorods as depicted in Fig. 7a occurs between substrateliquid interface and liquid-gas interface. The schematic sketch is illustrating each step in serial to have a better understanding of the process, but in reality the steps take place in parallel, so that a thin film with continuous reorientation from parallel to perpendicular nanorod orientation in several sublayers is processed as depicted in Fig. 3a.

In the intermediate regime the contact angle is reduced to a sufficiently low value that the contact line is pinned and a thin film is entrained on the substrate. Since the dried film is the thinnest as it is already known from Fig. 1, respectively the withdrawn wet film has to be also the thinnest. Consequently the substrate-near $\mathrm{ZnO}$ nanorods are highly impacted by shear force; simultaneously the undried wet film is so thin, that an evaporation driven reorientation of the nanorods is just possible on the outmost top layer in the given time, which results in a low surface order as seen in Fig. 2d. In the viscous drag 


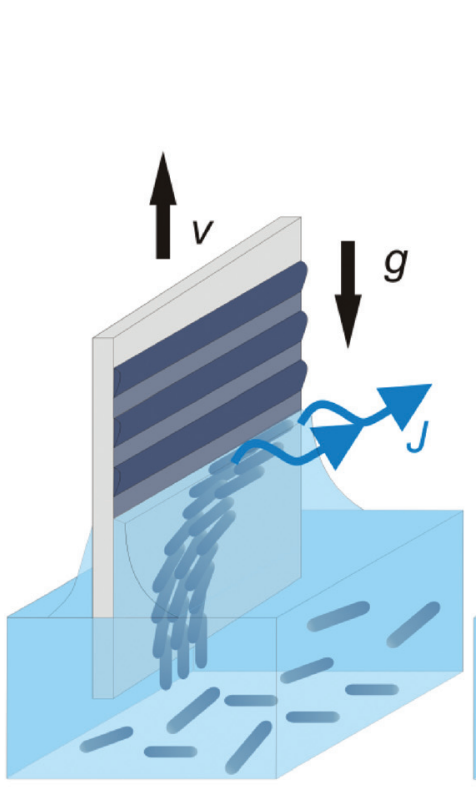

a) Capillary regime

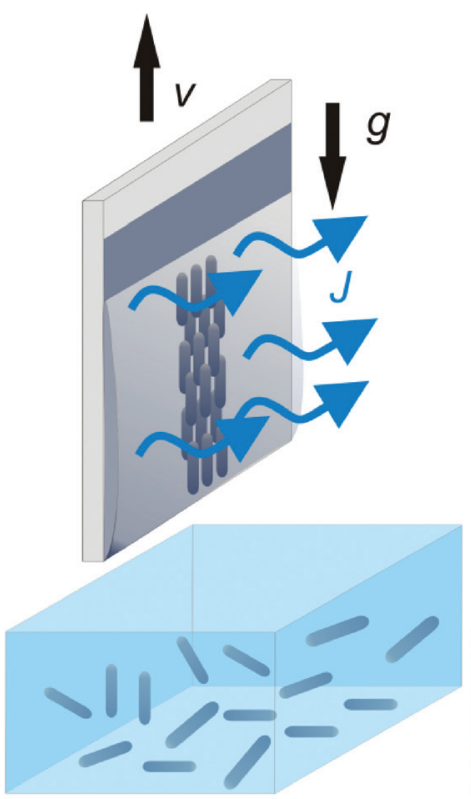

b) Intermediate regime
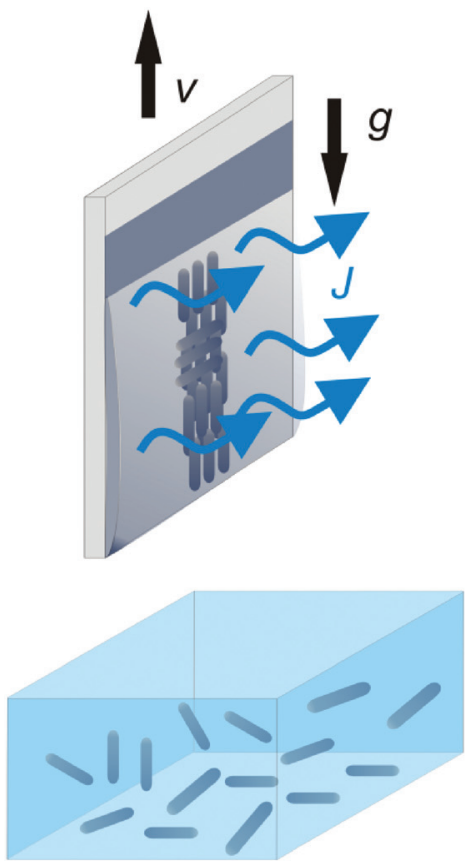

c) Viscous drag regime

Fig. 7 3D-sketch of the dip coating process and the structure formation mechanism in the capillary (a), intermediate (b) and viscous drag regime (c). The dried $\mathrm{ZnO}$ nanorod film above the wet film is based on the appearance in the light microscope. The withdrawal velocity $\mathrm{v}$, the gravity $\mathrm{g}$ and the evaporation flux $\mathrm{J}$ are acting on the nematic structure of the $\mathrm{ZnO}$ nanorod film.

regime the principal is approximately the same as in the intermediate regime, but the wet film is thicker compared to the intermediate regime. Consequently evaporation induced reorientation has a longer time scale. This results in domains or ensembles of $\mathrm{ZnO}$ nanorods on the surface, which are oriented perpendicular to the withdrawal direction. Due to the thicker wet film not just reorientation on the surface, but also in the bulk is still possible. Hence, less bulk order is achieved in the viscous drag regime compared to the intermediate regime.

The presented new insights into the dip-coating process of nanorods clearly show that understanding the film formation mechanism is mandatory for processing thin films for various optoelectronic applications. Knowledge about surface and bulk order is fundamental to utilize a functional layer structure in the thin film.

\section{Conclusions}

It was shown that nematic thin films with high quality are achieved by dip coating of $\mathrm{ZnO}$ nanorod dispersions in a controlled environment. Withdrawal regimes comparable to the processing of spherical particles could be identified, namely a viscous drag regime, an intermediate regime and a capillary regime. As shown by polarized Raman spectroscopy, quantification of the surface order, analysis of film thickness and roughness, processing of thin films in the different regimes, however, shows different structure formation mechanisms: the viscous drag regime as well as the intermediate regime is predominantly influenced by shear forces, which orient the nanorods in direction of withdrawal. The mechanism in the capillary regime is directed by shear forces superimposed by an evaporation-induced convective assembly. The stick-slip motion in the capillary regime induces high film roughness, however evaporation and reduction of the free Helmholtz energy results in high surface order. Relative high surface order, as well as bulk order, lowered roughness and thin films are gained in the viscous drag regime. Thinnest films with high bulk order are achieved in the intermediate regime.

By revealing the self-assembly mechanisms for nanorods, it is obvious that the combined analysis of surface and bulk is mandatory in nematic thin film characterization. From the Raman maps in the viscous drag, intermediate and capillary regime it is shown that polarized Raman spectroscopy gives access to bulk order in thin films made of anisotropic crystalline materials. Complementary information to bulk order characterization is given by SEM-based image analysis. Without invasive breaking of the substrate for a cross-sectional view, the overall order in the bulk can be analysed routinely.

\section{Acknowledgements}

The authors gratefully acknowledge funding of the German Research Council (DFG) through the Erlangen Research Training Group 1161 "Disperse Systems for Electronics" and 
research area A "Functional Particle Systems" in the Cluster of Excellence "Engineering of Advanced Materials" at the Friedrich-Alexander-Universität Erlangen-Nürnberg. The authors thank Prof. L. Frey and S. Polster at the Chair of Electron Devices at the University of Erlangen-Nürnberg for providing the substrates. The authors are also grateful to Prof. M. Guldi and A. Kunzmann at the Chair of Physical Chemistry at the University Friedrich-Alexander-Universität Erlangen-Nürnberg for providing help with film thickness measurements and T. Nacken at the Institute of Particle Technology for help and advice with Raman spectroscopy.

\section{References}

1 T. P. Niesen and M. R. de Guire, J. Electroceram., 2001, 6, 169-207.

2 P. T. Hammond, Adv. Mater., 2004, 16, 1271-1293.

3 M. Faustini, C. Boissière, L. Nicole and D. Grosso, Chem. Mater., 2014, 26, 709-723.

4 M. Grzelczak, J. Vermant, E. M. Furst and L. M. LizMarzán, ACS Nano, 2010, 4, 3591-3605.

5 B. A. Grzybowski, C. E. Wilmer, J. Kim, K. P. Browne and K. J. M. Bishop, Soft Matter, 2009, 5, 1110.

6 Evaporative Self-assembly of Ordered Complex Structures, ed. Z. Lin, World Scientific, Singapore, 2012.

7 S. Kinge, M. Crego-Calama and D. N. Reinhoudt, ChemPhysChem, 2008, 9, 20-42.

8 B. Sun and H. Sirringhaus, Nano Lett., 2005, 5, 2408-2413.

9 V. R. Dugyala, S. V. Daware and M. G. Basavaraj, Soft Matter, 2013, 9, 6711.

10 A. Dessombz, C. R. Pasquier, P. Davidson and C. Chanéac, J. Phys. Chem. C, 2010, 114, 19799-19802.

11 J. Wang, E. Khoo, P. S. Lee and J. Ma, J. Phys. Chem. C, 2008, 112, 14306-14312.

12 A. R. Tao, J. Huang and P. Yang, Acc. Chem. Res., 2008, 41, 1662-1673.

13 D. Grosso, J. Mater. Chem., 2011, 21, 17033.

14 M. Le Berre, Y. Chen and D. Baigl, Langmuir, 2009, 25, 2554-2557.

15 D. M. Kuncicky, R. R. Naik and O. D. Velev, Small, 2006, 2, 1462-1466.

16 S. P. Wargacki, B. Pate and R. A. Vaia, Langmuir, 2008, 24, 5439-5444.

17 W.-J. Chung, J.-W. Oh, K. Kwak, B. Y. Lee, J. Meyer, E. Wang, A. Hexemer and S.-W. Lee, Nature, 2011, 478, 364368.

18 L. Onsager, Ann. N. Y. Acad. Sci., 1949, 51, 627-659.

19 C. Kuemin, L. Nowack, L. Bozano, N. D. Spencer and H. Wolf, Adv. Funct. Mater., 2012, 22, 702-708.

20 S. Thiemann, M. Gruber, I. Lokteva, J. Hirschmann, M. Halik and J. Zaumseil, ACS Appl. Mater. Interfaces, 2013, 5, 1656-1662.

21 M. Mohammadimasoudi, L. Penninck, T. Aubert, R. Gomes, Z. Hens, F. Strubbe and K. Neyts, Opt. Mater. Express, 2013, 3, 2045.
22 B. Dan, A. W. K. Ma, E. H. Hároz, J. Kono and M. Pasquali, Ind. Eng. Chem. Res., 2012, 51, 10232-10237.

23 S. Schäfer, R. Srikantharajah, M. Klaumünzer, V. Lobaz, M. Voigt and W. Peukert, Thin Solid Films, 2014, 562, 659667.

24 J. Kim, J. Peretti, K. Lahlil, J.-P. Boilot and T. Gacoin, Adv. Mater., 2013, 25, 3295-3300.

25 J. L. Baker, L. H. Jimison, S. Mannsfeld, S. Volkman, S. Yin, V. Subramanian, A. Salleo, A. P. Alivisatos and M. F. Toney, Langmuir, 2010, 26, 9146-9151.

26 J. L. Baker, A. Widmer-Cooper, M. F. Toney, P. L. Geissler and A. P. Alivisatos, Nano Lett., 2010, 10, 195-201.

27 M. Zanella, R. Gomes, M. Povia, C. Giannini, Y. Zhang, A. Riskin, M. van Bael, Z. Hens and L. Manna, Adv. Mater., 2011, 23, 2205-2209.

28 D. Baranov, A. Fiore, M. van Huis, C. Giannini, A. Falqui, U. Lafont, H. Zandbergen, M. Zanella, R. Cingolani and L. Manna, Nano Lett., 2010, 10, 743-749.

29 B. Nikoobakht, Z. L. Wang and M. A. El-Sayed, J. Phys. Chem. B, 2000, 104, 8635-8640.

30 R. Marczak, F. Werner, R. Ahmad, V. Lobaz, D. M. Guldi and W. Peukert, Langmuir, 2011, 27, 3920-3929.

31 F. Hu, Y. Xia, Z. Guan, X. Yin and T. He, Electrochim. Acta, 2012, 69, 97-101.

32 H. Faber, J. Hirschmann, M. Klaumünzer, B. Braunschweig, W. Peukert and M. Halik, ACS Appl. Mater. Interfaces, 2012, 4, 1693-1696.

33 W. I. Park, G.-C. Yi, J.-W. Kim and S.-M. Park, Appl. Phys. Lett., 2003, 82, 4358.

34 M. Willander, O. Nur, Q. X. Zhao, L. L. Yang, M. Lorenz, B. Q. Cao, J. Zúñiga Pérez, C. Czekalla, G. Zimmermann, M. Grundmann, A. Bakin, A. Behrends, M. Al-Suleiman, A. El-Shaer, A. Che Mofor, B. Postels, A. Waag, N. Boukos, A. Travlos, H. S. Kwack, J. Guinard and D. Le Si Dang, Nanotechnology, 2009, 20, 332001.

35 B. Sun, R. Peterson, H. Sirringhaus and K. Mori, J. Phys. Chem. C, 2007, 111, 18831-18835.

36 Y. Li and Y. Wu, J. Am. Chem. Soc., 2009, 131, 5851-5857.

37 J. M. Russell, S. Oh, I. LaRue, O. Zhou and E. T. Samulski, Thin Solid Films, 2006, 509, 53-57.

38 J. Huang, R. Fan, S. Connor and P. Yang, Angew. Chem., Int. Ed., 2007, 119, 2466-2469.

39 M. Voigt, M. Klaumünzer, H. Thiem and W. Peukert, J. Phys. Chem. C, 2010, 114, 6243-6249.

40 J. Li, B. Cabane, M. Sztucki, J. Gummel and L. Goehring, Langmuir, 2012, 28, 200-208.

41 G. Berteloot, A. Daerr, F. Lequeux and L. Limat, Chem. Eng. Process., 2013, 68, 69-73.

42 M. Ghosh, F. Fan and K. J. Stebe, Langmuir, 2007, 23, 21802183.

43 H. Bodiguel, F. Doumenc and B. Guerrier, Langmuir, 2010, 26, 10758-10763.

44 S. Watanabe, K. Inukai, S. Mizuta and M. T. Miyahara, Langmuir, 2009, 25, 7287-7295.

45 H. Li, T. C. Hain, A. Muzha, F. Schöppler and T. Hertel, ACS Nano, 2014, 8, 6417-6424. 
46 E. Rio, A. Daerr, F. Lequeux and L. Limat, Langmuir, 2006, 22, 3186-3191.

47 R. Loudon, Adv. Phys., 1964, 13, 423-482.

48 I. Zardo, S. Conesa-Boj, F. Peiro, J. R. Morante, J. Arbiol, E. Uccelli, G. Abstreiter and A. Fontcuberta i Morral, Phys. Rev. B: Condens. Matter Mater. Phys., 2009, 80(24), 245324.

49 H. M. Fan, X. F. Fan, Z. H. Ni, Z. X. Shen, Y. P. Feng and B. S. Zou, J. Phys. Chem. C, 2008, 112, 1865-1870.

50 C.-L. Hsiao, L.-W. Tu, T.-W. Chi, M. Chen, T.-F. Young, C.-T. Chia and Y.-M. Chang, Appl. Phys. Lett., 2007, 90, 43102.
51 C.-T. Chien, M.-C. Wu, C.-W. Chen, H.-H. Yang, J.-J. Wu, W.-F. Su, C.-S. Lin and Y.-F. Chen, Appl. Phys. Lett., 2008, 92, 223102.

52 J. L. Yu, Y. F. Lai, Y. Z. Wang, S. Y. Cheng and Y. H. Chen, J. Appl. Phys., 2014, 115, 33505.

53 Zinc Oxide. Fundamentals, Materials and Device Technology, ed. M. Hadis and Ö. Ümit, Wiley-VCH Verlag $\mathrm{GmbH} \& \mathrm{Co}$. KGaA, Weinheim, Germany, 2009.

54 R. Zhang, P.-G. Yin, N. Wang and L. Guo, Solid State Sci., 2009, 11, 865-869.

55 M. Rajalakshmi, A. K. Arora, B. S. Bendre and S. Mahamuni, J. Appl. Phys., 2000, 87, 2445. 